\title{
Advantages of natural microcrystalline graphite filler over petroleum coke in isotropic graphite preparation
}

\author{
Ke Shen ${ }^{a, b}$, Zheng-Hong Huang ${ }^{a}$, Kaixin Hu ${ }^{c}$, Wanci Shen ${ }^{a}$, Suyuan $\mathrm{Yu}^{d}$, \\ Junhe Yang ${ }^{e}$, Guangzhi Yang ${ }^{e}$, Feiyu Kang ${ }^{a, f, *}$ \\ a State Key Laboratory of New Ceramics and Fine Processing, School of Materials Science and Engineering, Tsinghua University, \\ Beijing 100084, China \\ ${ }^{\mathrm{b}}$ Institute of Nuclear and New Energy Technology, Tsinghua University, Beijing 100084, China \\ c National Microgravity Laboratory, Institute of Mechanics, Chinese Academy of Sciences, Beijing 100190, China \\ d Center for Combustion Energy, Department of Thermal Engineering, Tsinghua University, Beijing 100084, China \\ e School of Materials Science and Engineering, University of Shanghai for Science and Technology, Shanghai 200093, China \\ ${ }^{f}$ City Key Laboratory of Thermal Management Engineering and Materials, Graduate School at Shenzhen, Tsinghua University, \\ Shenzhen 518055, Guangdong, China
}

\section{A R T I C L E I N F O}

Article history:

Received 9 December 2014

Accepted 26 March 2015

Available online 1 April 2015

\begin{abstract}
A B S T R A C T
Graphite materials are used in high-temperature gas-cooled reactor (HTR) as moderator and reflector. Nuclear graphite is currently manufactured using coke as the filler; however, improvements in the performance of nuclear graphite are anticipated in order to enhance the safety and lifetime of HTRs. Natural microcrystalline graphite (MG), which is a metamorphic product of coal, is an emerging candidate as filler material. This paper proposes the approach of preparing isotropic graphite using MG fillers. The characterization results of MG ores and purified powder indicate a polycrystalline and near-isotropic structure of MG particles. Thus, near-isotropic graphite with an isotropy ratio of 1.10-1.15 can be easily obtained via cold isostatic pressing. The thermal diffusivity of MG-based green body is much higher than that of coke-based one, therefore facilitates the baking step. The advantages of MG-based graphite also include a high degree of graphitization, and a low coefficient of thermal expansion, both of which are highly beneficial to nuclear applications. As a result, MG exhibits a large potential for the application in isotropic nuclear graphite.
\end{abstract}

(c) 2015 Elsevier Ltd. All rights reserved.

\section{Introduction}

The moderator in a nuclear reactor thermalizes $2 \mathrm{MeV}$ fast neutrons to $0.025 \mathrm{eV}$ thermal neutrons. With low atomic weight, high moderating ratio and low absorption cross section, $\mathrm{H}_{2} \mathrm{O}, \mathrm{D}_{2} \mathrm{O}, \mathrm{Be}$, and $\mathrm{C}$ are four possible moderators [1]. Carbon in the form of graphite offers an acceptable compromise between nuclear properties and cost, therefore graphite is employed as moderator and structural material in high-temperature gas-cooled reactors (HTR) $[2,3]$.

\footnotetext{
* Corresponding author at: State Key Laboratory of New Ceramics and Fine Processing, School of Materials Science and Engineering, Tsinghua University, Beijing 100084, China. Tel./fax: +86106279 2911.

E-mail address: fykang@mail.tsinghua.edu.cn (F. Kang). 
Major processing steps in the manufacture of polycrystalline graphite are briefly described as follows [4]: calcined coke powder is blended with coal-tar pitch. The pitch binder plasticizes the coke filler particles so that they can be formed. Extrusion, vibration, and isostatic pressing are commonly used forming processes. The green bodies are baked at roughly $1000^{\circ} \mathrm{C}$, and then pitch-impregnated to increase the bulk density. Impregnation and rebake may occur several times to attain the required density. The final stage is graphitization at $2500-3000^{\circ} \mathrm{C}$, during which the carbon atoms rearrange to form a ordered graphite lattice. Additionally, a halogen purification stage is included to achieve nuclear-grade chemical purity.

The graphite reflector in a pebble-bed design HTR is a nonreplaceable component during the reactor's lifetime. The behavior of nuclear graphite under fast neutron irradiation, therefore, directly influences the reactor's lifetime [5]. The reactor performance will be enhanced by improving the irradiation property of the graphite.

The properties of polycrystalline graphite strongly depend on the texture of the graphite [6], which in turn depends on the structure of the filler particles [7], and thereby can be modified by the introduction of new filler materials.

Current commercialized nuclear graphite is produced from various coke types, including needle coke, Gilsonite coke and isotropic coke. Needle coke was used for early nuclear graphite such as Pile Grade A (PGA) [8]. Gilsonite coke is obtained from a naturally occurring pitch found at Bonanza in Utah in the United States [9]. Isotropic cokes with various textures are derived from either oil derivatives (petroleum cokes) or coaltar pitch (pitch cokes).

Natural microcrystalline graphite (MG) is a promising alternative. Natural occurrence of graphite is found widely throughout the world. It is formed by the metamorphosis of sediments containing carbonaceous material. Natural graphite is considered, on the basis of physical appearance, to fall into three principal types: flake graphite, microcrystalline graphite and lump graphite. Natural MG is an aggregate of randomly orientated graphite micro-crystallites $[10,11]$, the ore in bulk has a very close appearance to coal, as shown in Fig. 1(b). It is easily crushed into powder by milling. Fig. 1(c) shows a typical powder XRD pattern with sharp (002) diffraction, indicating high crystallization of the graphite structure, even in MG ores. Table 1 lists the carbon content and crystal parameters of natural MG. Carbon content ranging from 75 to $90 \mathrm{wt} . \%$ is measured for the major Chinese natural MG ores, and the degree of graphitization is usually over $90 \%$.

There are large and widespread deposits of natural MG in China, as shown in Fig. 1(a). The largest mine, with a reserve of 30 million tons, is located in the Lutang area of Chenzhou, Hunan Province. Other major deposits are known in the Provinces of Inner Mongolia (Bayan Nur), Hubei (Shiyan), Fujian (Sanming), Jilin (Panshi), and Heilongjiang (Yichun). The utilization of MG is currently confined to a role as primary level such as raw materials for lead in pencil, crucible, and carburant for steel making. Investigations into further applications of MG-based graphite will greatly promote the development of the MG industry in China.
For nuclear application, natural graphite especially flake graphite has been successfully used in HTR spherical fuel elements, the graphite matrix of which is manufactured using a powder mixture composed of $64 \mathrm{wt} . \%$ natural flake graphite, 16 wt.\% artificial graphite, and 20 wt.\% phenol resin binder $[12,13]$. With such fuel elements the AVR reactor has been successfully operated for 21 years.

In this study, we demonstrate that MG exhibits a large potential for the application in isotropic nuclear graphite. The structures of MG ore and purified MG were characterized, and a comparative study of MG and petroleum coke was conducted in order to evaluate the properties of MG-based graphite.

\section{Experimental}

\subsection{Filler materials}

Natural MG ore from Chenzhou, Hunan Province, China, was selected as the raw material. Graphite ores with approximately 80 wt.\% carbon were purified to 99.9 wt.\% carbon by a high temperature purification method. Purified MG powders with $D_{50}$ values (particle diameter at $50 \%$ in the cumulative particle size distribution) of 48.1 and $17.1 \mu \mathrm{m}$ were mixed in a mass ratio of 3:1, respectively. A 325 mesh petroleum coke powder with a $D_{50}$ of $38.2 \mu \mathrm{m}$ was used to give a comparative analysis. Mesocarbon microbeads (MCMBs) with a $D_{50}$ of $25.0 \mu \mathrm{m}$ were also included in some individual tests to provide more context for the results.

The mixed-MG and petroleum coke powders have similar particle size distributions, as shown in Fig. 2(a), which was obtained by laser a diffraction particle size analyzer; therefore, the two powders are comparable in forming, baking, and in the physical properties of final product. The tapped densities of mixed-MG and petroleum coke powders are 0.89 and $1.06 \mathrm{~g} \mathrm{~cm}^{-3}$, respectively. Both powders were kneaded with 30 wt.\% coal-tar pitch, as shown in Fig. 2(b).

\subsection{Isotropic graphite sample preparation}

The green powders were formed by cold isostatic pressing at $200 \mathrm{MPa}$, in a cylindrical rubber mold of height $80 \mathrm{~mm}$ and inner diameter $80 \mathrm{~mm}$. The green bodies had a diameter of approximately $70 \mathrm{~mm}$ and a height of approximately $70 \mathrm{~mm}$, and were baked at $1000{ }^{\circ} \mathrm{C}$ in flowing $\mathrm{N}_{2}$. Impregnation and rebake, which increased the bulk density to over $1.70 \mathrm{~g} \mathrm{~cm}^{-3}$, were conducted at Sinosteel Advanced Materials (Zhejiang) Co., Ltd. Graphitization was carried out in Ar gas in an intermediate-frequency induction heating furnace. The samples were first heated with a constant power of $5 \mathrm{~kW}$ to $1000^{\circ} \mathrm{C}$, and then at a rate of $10^{\circ} \mathrm{C} \mathrm{min} \mathrm{m}^{-1}$, up to $2800^{\circ} \mathrm{C}$. After a holding time of $1 \mathrm{~h}$, the furnace chamber was cooled naturally to room temperature. Photographs of the MG-based green body, baked, and graphitized artifacts are shown in Fig. 3.

\subsection{Green powder compression test}

Green powders produced from MG and coke, as well as MCMBs, were compacted in a Toni Technik compression machine. $5.0 \mathrm{~g}$ powder was placed into a steel die with an 
(a)

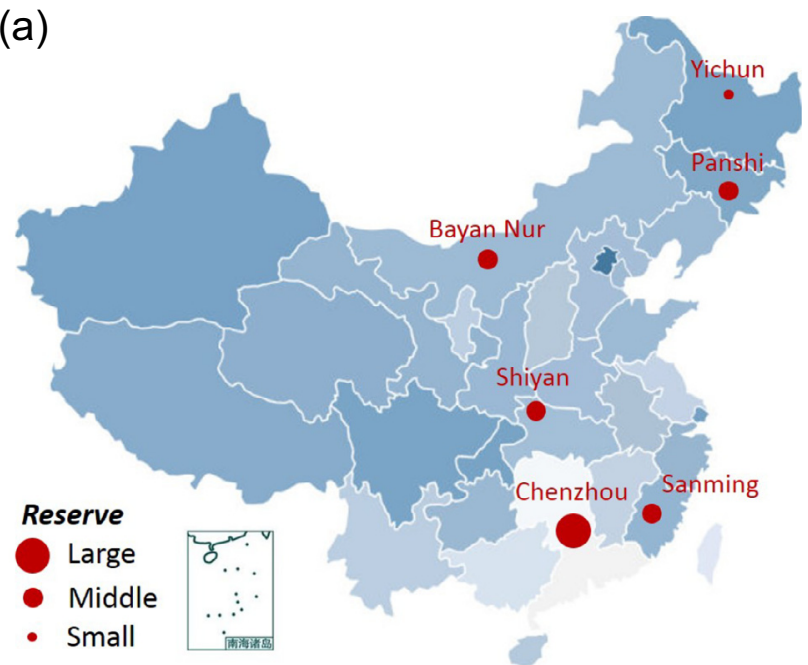

(b)

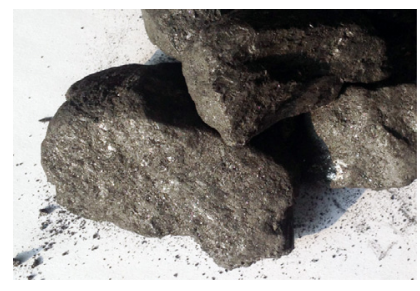

(c)

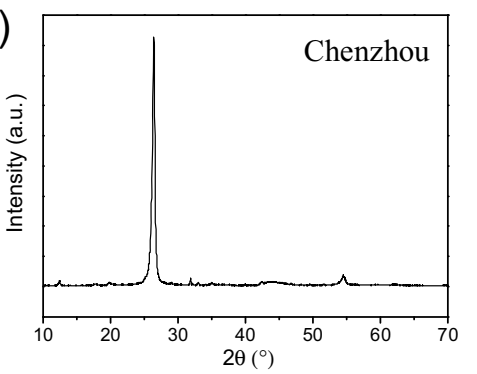

Fig. 1 - (a) Distribution of natural microcrystalline graphite in China, (b) photograph of ore from Bayan Nur, (c) powder XRD pattern of natural microcrystalline graphite ore from Lutang of Chenzhou. (A color version of this figure can be viewed online.)

\begin{tabular}{|c|c|c|c|c|}
\hline Place of origin & C (wt.\%) & $d_{002}(n m)$ & Graphitization degree (\%) & (002) FWHM $\left(^{\circ}\right)$ \\
\hline Lutang, Chenzhou & 86.7 & 0.3364 & 88.8 & 0.379 \\
\hline Linwu, Chenzhou & 79.4 & 0.3358 & 95.3 & 0.370 \\
\hline Bayannur & 84.6 & 0.3359 & 94.2 & 0.300 \\
\hline Panshi & 75.2 & 0.3359 & 94.2 & 0.319 \\
\hline Sanming & 86.5 & 0.3362 & 91.0 & 0.370 \\
\hline
\end{tabular}
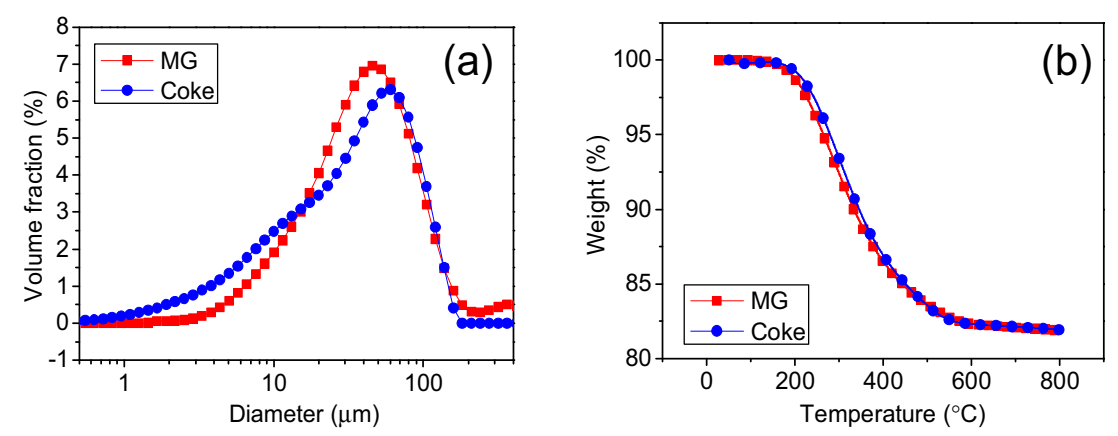

Fig. 2 - (a) Particle size distributions of purified mixed-MG and petroleum coke filler. (b) TG curves of green powders. (A color version of this figure can be viewed online.)
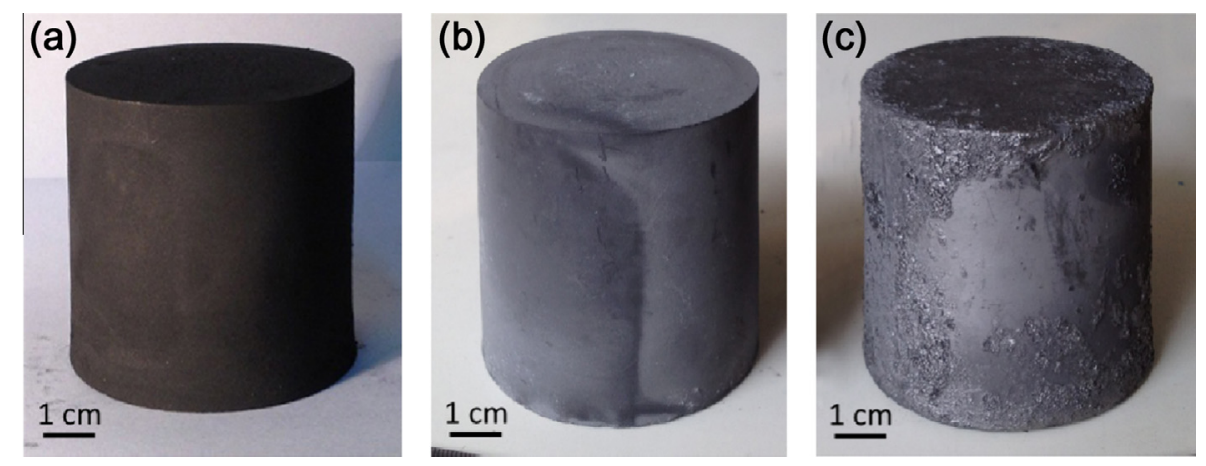

Fig. 3 - Natural MG-based graphite. (a) Green body, (b) baked body, (c) final product after impregnation and graphitization. (A color version of this figure can be viewed online.) 
inner diameter of $25.00 \mathrm{~mm}$. The powder was pre-compressed at $40 \mathrm{MPa}$, and then pressed to $220 \mathrm{MPa}$ at a rate of $5 \mathrm{MPa} \mathrm{s}^{-1}$. The pressure was released after holding for $10 \mathrm{~s}$. The relationship between the sample height and pressure was recorded as raw data, and was converted to a density-pressure relationship during both compression and decompression.

\subsection{Dimensional change}

The volume change of MG and coke green bodies during baking was measured. About $3 \mathrm{~g}$ of green powder was die-molded and then isostatically pressed at $200 \mathrm{MPa}$ into a small pellet with a diameter of $22 \mathrm{~mm}$. These green bodies were heated to designated temperatures in nitrogen at a rate of $5^{\circ} \mathrm{C} \mathrm{min}^{-1}$ without holding. After cooling, sample mass was recorded, density of the specimens was measured by Archimedes' principle, and volume change of specimens was determined by the weight loss and the bulk density.

\subsection{Thermal diffusivity}

For this test, the pellet-shaped green bodies were baked by heating to designated temperatures in nitrogen at a rate of $5^{\circ} \mathrm{C} \mathrm{min}^{-1}$, and held for $1 \mathrm{~h}$. The baked bodies were cut into appropriate dimensions.

Thermal diffusivity was measured using the laser flash method with a Netzsch Laser Flash Apparatus (LFA 447 MicroFlash) under in-plane mode [14,15]. The measuring followed ASTM standard E1461-07 [16].

\subsection{Coefficient of thermal expansion and isotropy ratio}

Isotropy ratio is defined as the ratio of the coefficient of thermal expansion (CTE) in the against-grain direction to its corresponding value in the with-grain direction. The dimensions of the specimens were $5 \times 5 \times 25 \mathrm{~mm}$. A thermal dilatometer (NETZSCH DIL 402 PC) was used to measure CTE in the temperature range of $20-400^{\circ} \mathrm{C}$, with a heating rate of $10^{\circ} \mathrm{C} \min ^{-1}$.

\section{Results and discussions}

\subsection{Natural MG}

\subsubsection{Ores}

MG, also known as amorphous graphite, is made up of aggregates of fine graphite crystals less than $1 \mu \mathrm{m}$ in size. MG is formed by the thermal metamorphosis of coal [17].

The microcrystalline nature of natural MG is seen in the fracture surface of a graphite ore particle (Fig. 4), where individual graphite crystallites are clearly visible. Graphite micro-crystallites less than $500 \mathrm{~nm}$ in size are arranged with random orientations, eliminating a preferred orientation and helping to give MG particles their near-isotropic property.

\subsubsection{Purification}

To obtain high purity natural graphite, chemical purification using either $\mathrm{NaOH}$ or a mixed solution of $\mathrm{HCl}$ and $\mathrm{HF}$ is commonly used, in particular with flake graphite. However, it is less satisfactory for MG since the impurities are dispersed throughout the ores and mixed together with graphite micro-crystallites. Recently, a high temperature purification method has been employed by some Chinese factories. The equipment can be described as a vertically-installed tube furnace, with isostatic graphite blocks used to build the interior structure. Crucibles containing MG ore powders are fed into the furnace from the top, and pass through the high temperature region, which is inductively heated to $3000{ }^{\circ} \mathrm{C}$ in an inert atmosphere. The feedstock input and output are a continuous operation.

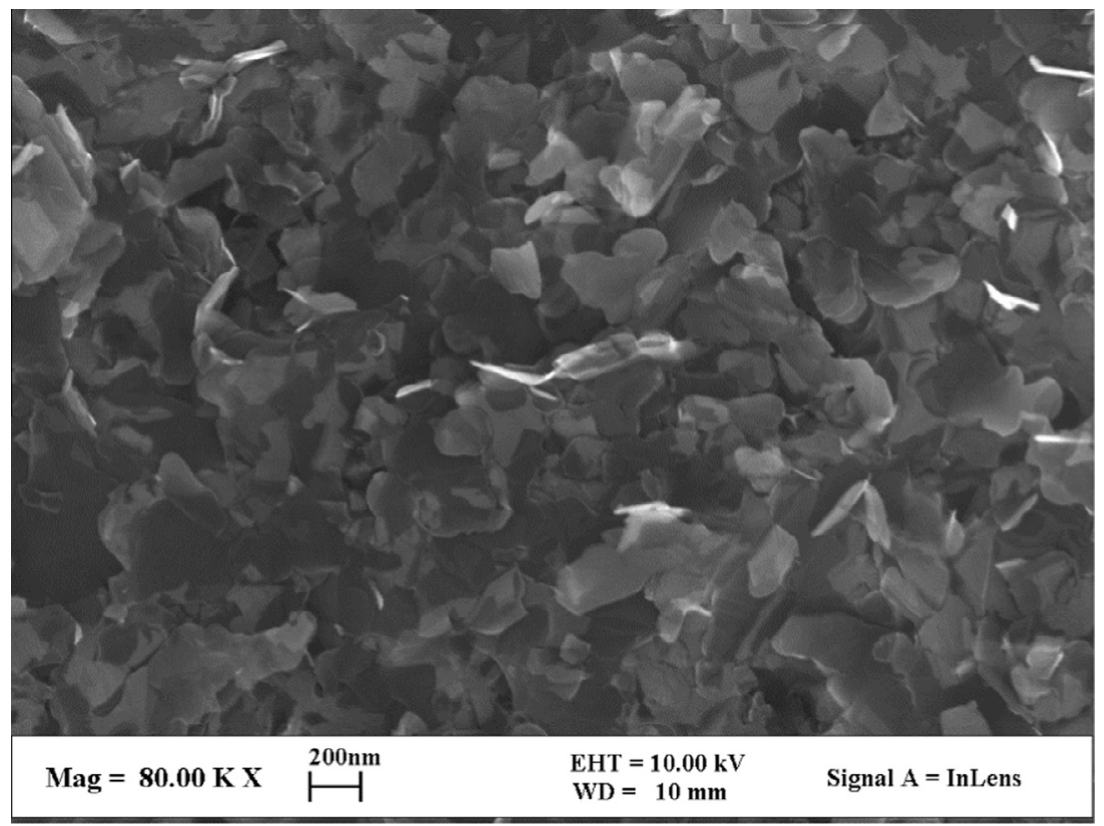

Fig. 4 - Microstructure of MG ore. 

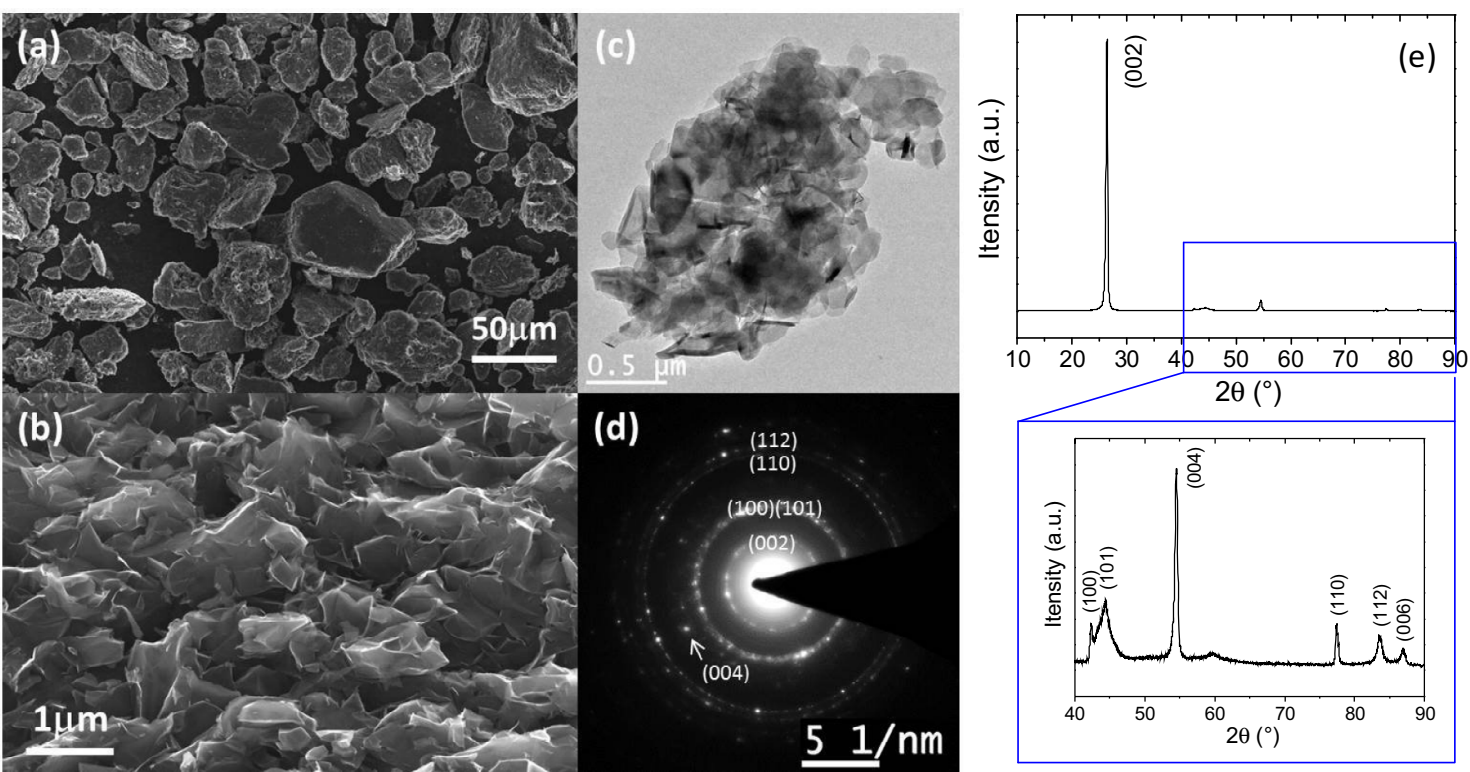

Fig. 5 - High temperature purified natural MG. (a) Particle morphology, (b) SEM image of fracture surface, (c) TEM image of an individual MG particle, (d) selected area electron diffraction pattern, (e) XRD pattern of purified MG powder. The inset below is a zoomed-in XRD pattern for $2 \theta=40-90^{\circ}$. (A color version of this figure can be viewed online.)

The high temperature purified MG can reach 99.9 wt.\% C in regular operation. Fig. 5 shows the morphology and microstructure of high temperature purified MG particles. The purified MG is ground to produce isometric particles, as shown in Fig. 5(a). Graphite micro-crystallites coalesce to form larger graphite sheets with a lateral dimension of about $1 \mu \mathrm{m}$, as shown in Fig. 5(b). Fig. 5(c, d) shows the TEM image and selected area electron diffraction pattern. The appearance of diffraction rings of (002), (100), (101), (110), (112) indicates that purified MG particles have typical polycrystalline structure. The powder XRD pattern of purified MG is presented in Fig. 5(e). Comparing with its ore (Table 1), the $d_{002}$ value decreases to $0.3361 \mathrm{~nm}$, the degree of graphitization is $91.7 \%$ accordingly, and the (002) FWHM decreased to $0.182^{\circ}$ due to crystal growth.

\subsection{Dimensional and bulk density change during baking}

Fig. 6 displays the volume change of green bodies during baking. A remarkable difference in the dimensional change is found between MG-based and coke-based green bodies. The MG-based specimen undergoes a volume expansion of around $7 \%$ when heated to $200^{\circ} \mathrm{C}$, whereas the petroleum coke-based specimen keeps shrinking from the outset. After baking, a slight net expansion is found for the MG-based specimen. Such an initial expansion at $200^{\circ} \mathrm{C}$ is attributed to relaxation of the internal stresses that were generated during forming.

Accordingly, the initial expansion of MG-based specimen can be reflected in a bulk density change, as shown in Fig. 6. The MG-based green body shows higher density than

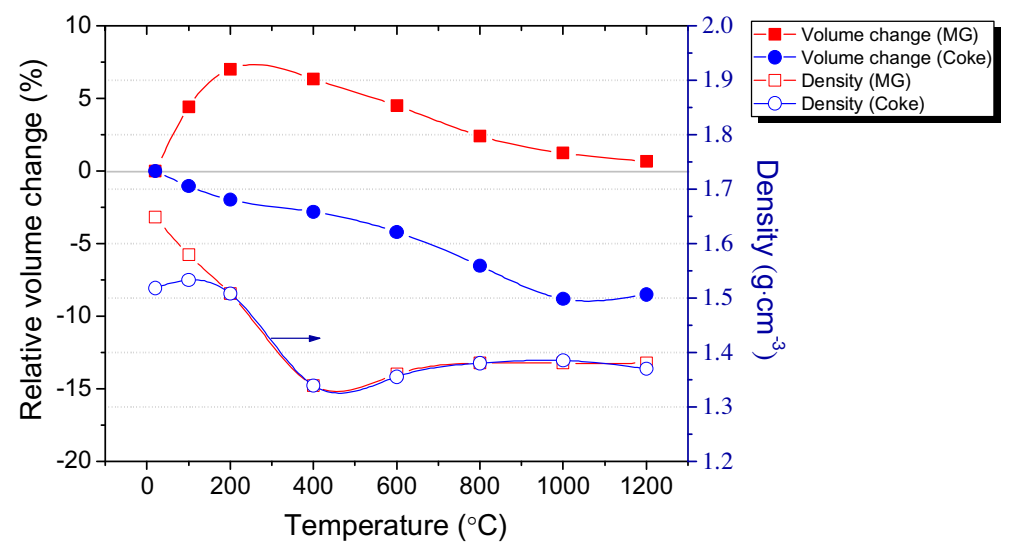

Fig. 6 - Relative volume change and density of green bodies during baking. (A color version of this figure can be viewed online.) 


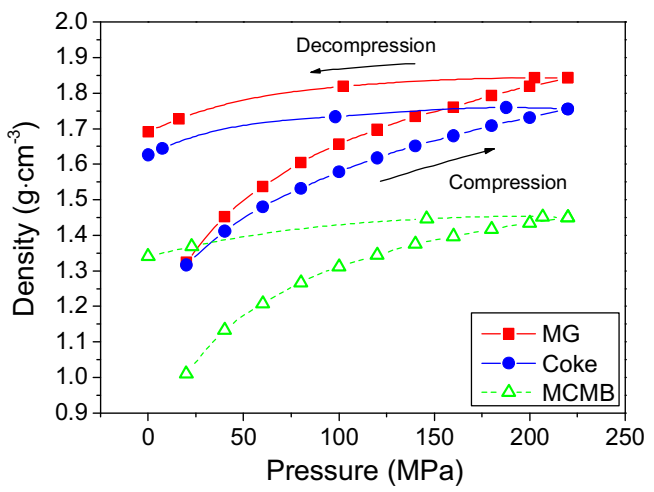

Fig. 7 - Compression and decompression behavior of MGbased and petroleum coke-based green powders and MCMBs. (A color version of this figure can be viewed online.)

coke-based one, however, the initial expansion remarkably decreases the density at $200^{\circ} \mathrm{C}$, after which the MG-based and coke-based green bodies show almost identical density.

The difference in bulk density after isostatic pressing is explained in terms of powder compression behavior. Fig. 7 shows the relationship between bulk density and pressure during compression and decompression for MG and coke green powders and MCMB. The densification of green powders (elimination of pores), in addition to elastic deformation, results in a density increase during compression. Comparing the MG and petroleum coke, the density of green bodies based on MG becomes greater than those based on petroleum coke at higher pressures. During decompression, a slight decrease of bulk density occurs owing to the release of elastic deformations. The bulk densities of MG-based and coke-based green bodies are 1.69 and $1.63 \mathrm{~g} \mathrm{~cm}^{-3}$, respectively, indicating that MG powder is easier to compress. One possible explanation may be that low surface friction of MG facilitates the rearrangement of particles to achieve better densification. MCMBs are a spherical carbonaceous precursor; the green body derived from MCMBs shows much lower density than for MG and petroleum coke.

\subsection{Mechanical properties}

The bulk densities of MG-based and coke-based graphite are shown in Table 2. The coke-based green body of approximately $70 \mathrm{~mm}$ diameter has a density of $1.57 \mathrm{~g} \mathrm{~cm}^{-3}$, whereas the MG-based one has a higher density of $1.66 \mathrm{~g} \mathrm{~cm}^{-3}$, which is explained in terms of powder compression behavior. After baking, the two carbon specimens have the same densities of $1.42 \mathrm{~g} \mathrm{~cm}^{-3}$. Increase in density was achieved by two cycles of pitch impregnation and rebake. Graphitization at $2800^{\circ} \mathrm{C}$ did not have an effect on the density of MG-based carbon, whereas for coke-based carbon, the density increased as a result of shrinkage of the coke filler.

For samples prepared in the same manner, MG-based and coke-based graphite show flexural strength of 22.9 and 17.4 MPa, respectively. This demonstrates that MG-based graphite is quite comparable in strength to graphite produced from coke.

\subsection{Advantages of natural MG filler}

\subsubsection{High thermal diffusivity of green body}

Green bodies produced from natural MG have a distinct advantage during baking, as a result of their higher thermal diffusivity. From the heat conduction equation:

$\frac{\partial \mathrm{T}}{\partial \mathrm{t}}=\alpha \nabla^{2} \mathrm{~T}$

thermal diffusivity $\alpha$ is a measure of how quickly a body can change its temperature. Green bodies with higher thermal diffusivity will more rapidly approach a homogenous temperature distribution, which is very beneficial to carbon baking. The thermal diffusivity of carbon specimens at designated temperatures during baking is shown in Fig. 8. Several features of these curves are of interest:

(1) Produced from MG filler that is already well-graphitized, MG-based green bodies have much higher thermal diffusivity than petroleum coke-based and MCMB-based green bodies throughout their carbonization. As a result, temperature gradients and thermal stress in artifacts are significantly reduced. The temperature difference between surface and center of an artifact can be calculated by using the heat conduction equation and the temperature-dependent thermal diffusivity in Fig. 8. For an artifact of $70 \mathrm{~mm}$ diameter and $70 \mathrm{~mm}$ height, and a constant heating rate of $3{ }^{\circ} \mathrm{C} \mathrm{min}^{-1}$, the result is shown in Fig. 9. The temperature difference in the MG-based artifact is only onefourth that in the coke-based artifact.

(2) The thermal diffusivities show an increase at elevated temperature, as the pitch binder is transforming to coke. It is known that aromatic condensation reactions occur at temperatures of $500-1000^{\circ} \mathrm{C}$ ) [18], and lead to formation of the final residual coke, thereby causing enhancement of thermal diffusivity.

(3) The MCMB carbon shows much lower thermal diffusivity, which is a key reason causing green bodies to crack. After heat treatment at $1000^{\circ} \mathrm{C}$, the coke filler and

Table 2 - Mechanical properties of MG-based and coke-based graphite.

\begin{tabular}{llrr} 
& & MG & Coke \\
\hline Density $\left(\mathrm{g} \mathrm{cm}^{-3}\right)$ & Green body & 1.66 & 1.57 \\
& Baked & 1.42 & 1.42 \\
& Impregnated and rebaked & 1.78 & 1.77 \\
Flexural strength $(\mathrm{MPa})$ & Graphitizated & 1.78 & 1.83 \\
\hline
\end{tabular}




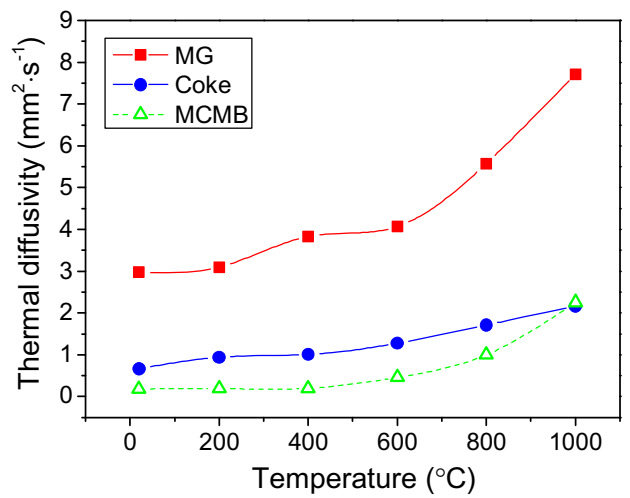

Fig. 8 - Thermal diffusivity $\alpha$ of green bodies during baking. (A color version of this figure can be viewed online.)

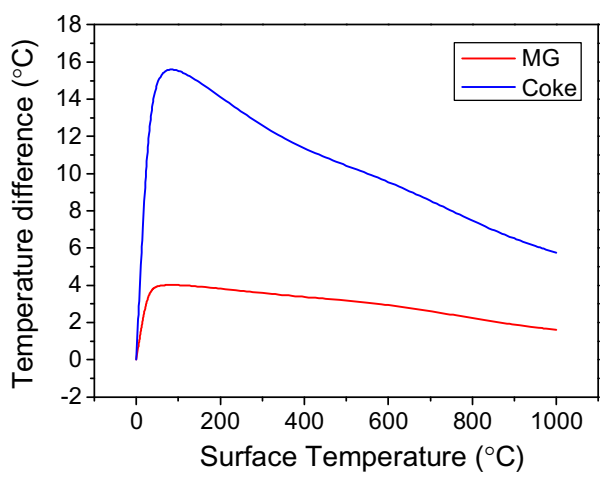

Fig. 9 - The temperature difference between surface and center of artifact $(\Phi 70 \mathrm{~mm} \times 70 \mathrm{~mm})$ with a constant heating rate of $3^{\circ} \mathrm{C} \mathrm{min}^{-1}$. (A color version of this figure can be viewed online.)

binder phases in petroleum coke carbon, and the mesophase derived coke, are all turbostratic carbons. Thus, the thermal diffusivities are similar in these two carbons.

\subsubsection{High graphitization degree}

Radiation damage in graphite by fast neutrons creates lattice vacancies and interstitial carbon atoms. A principal result of introducing defects is crystallite dimensional change: interstitial defects will cause crystallite growth in the 'c' direction, whereas coalescence of vacancies will cause shrinkage in the ' $a$ ' direction $[1,6,19]$. These mechanisms of dimensional change are reflected in the behavior of polycrystalline graphite [7]. The texture of graphite, in particular the Mrozowski cracks formed during manufacture, is another important factor affecting dimensional change in polycrystalline graphite. Microcracks aligned parallel to the graphite layer plane initially accommodate the ' $c$ ' direction expansion of graphite crystallites, so primarily ' $a$ ' direction shrinkage will be observed. Graphite thus undergoes an initial bulk shrinkage at low neutron dose. With increasing dose, the shrinkage rate reduces until the graphite begins to expand or 'turns around', associated with closure of the Mrozowski cracks [7]. Generation of new cracks and pores eventually causes total disintegration of the graphite. The lifetime of nuclear graphite is typically defined by the neutron dose at which it returns to its original dimensions [20,21].

Nuclear graphite with a high degree of graphitization has a lower initial number of lattice defect sites available to trap irradiation-induced vacancies, and thus the rate of damage accumulation is reduced [4]. Therefore, increased graphitization temperature delays the turn-around, and subsequent

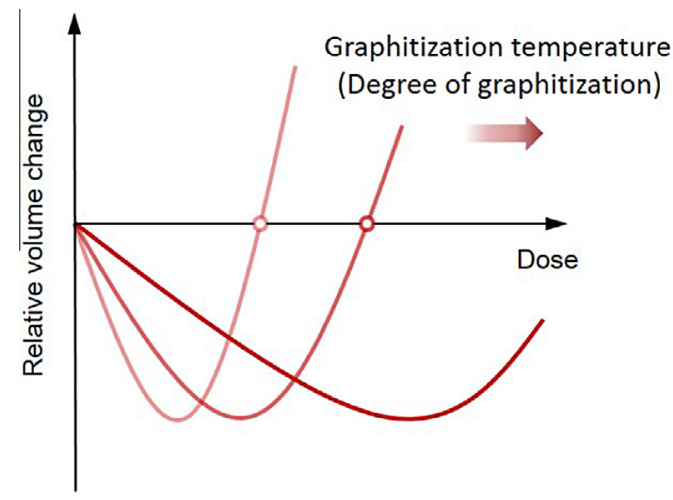

Fig. 10 - Dimensional stability of the same graphite, for heat treatment at different temperatures. (A color version of this figure can be viewed online.)

Table 3 - Lattice constants, crystallite sizes, and degree of graphitization of MG-based graphite, IG-110, and NBG-18.

\begin{tabular}{llllll} 
& $d_{002}(n m)$ & $L_{\mathrm{c}}(\mathrm{nm})$ & $L_{\mathrm{a}}(\mathrm{nm})$ & $g(\%)$ & Refs. \\
\hline MG & 0.3365 & 33.2 & 55.3 & 87.2 & This study \\
IG-110 & 0.3368 & 32.4 & 39.6 & 83.7 & This study \\
& $0.3364^{*}$ & 27.1 & 12.1 & 88.0 & {$[23]$} \\
& 0.3367 & - & - & 84.8 & {$[24]$} \\
NBG-18 & 0.3372 & 31.8 & 43.4 & 79.1 & This study \\
& $0.3369^{*}$ & 26.1 & 9.5 & 82.7 & {$[23]$} \\
& 0.3368 & - & - & 83.5 & {$[24]$} \\
\hline
\end{tabular}

These value were determined by plotting $d=n k /(2 \sin \theta)$ vs. $x=[\cos 2 \theta / \sin \theta+\cos 2 \theta / \theta] / 2$ and extrapolating as $x$ approaches 0 .

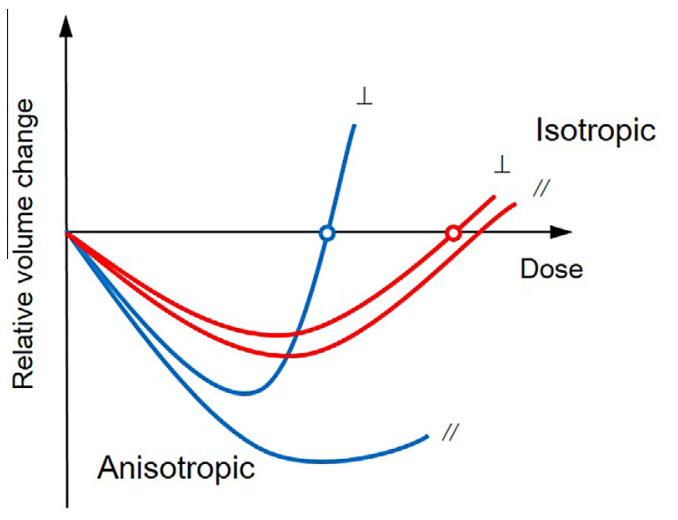

Fig. 11 - Dimensional stability of isotropic and anisotropic graphite. $\perp$ indicates against-grain, // indicates with-grain. (A color version of this figure can be viewed online.) 
(a)

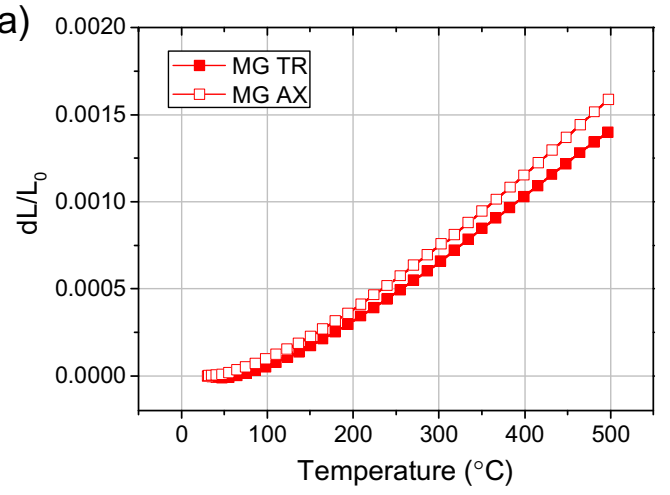

(b)

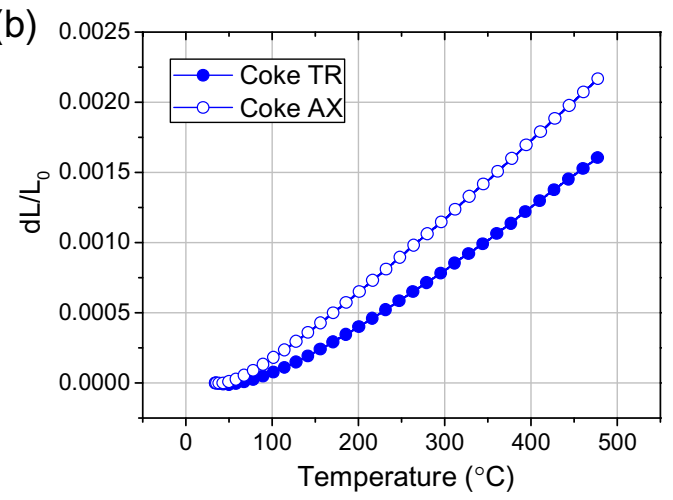

(c)

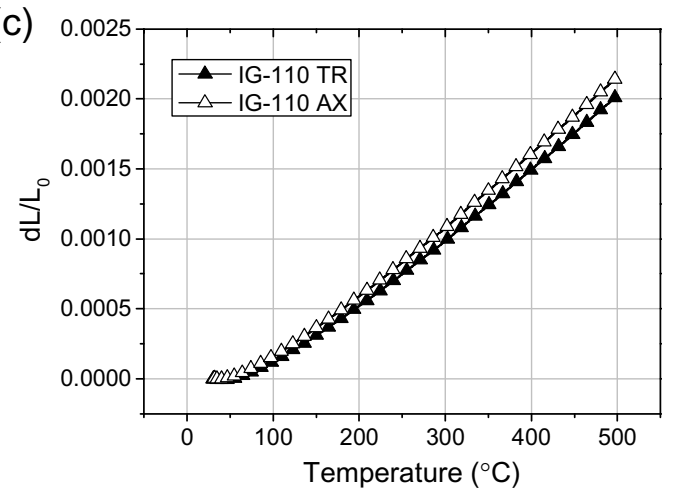

Fig. 12 - CTE curves of (a) MG-based graphite, (b) petroleum coke-based graphite, (c) IG-110. (A color version of this figure can be viewed online.)

dimensional growth is at a slower rate [22], as illustrated in Fig. 10.

The MG-based graphite shows a higher degree of graphitization (87.2\%) and larger crystallite size than the commercial nuclear graphites IG-110 and NBG-18. The $d_{002}$ value in this study was measured by the position of the (002) peak. IG-110 and NBG-18 show degrees of graphitization of $83.7 \%$ and $79.1 \%$, respectively. Zheng et al. [23] and Chi et al. [24] also reported the degree of graphitization for IG-110 and NBG-18, as shown in Table 3; in those two studies, IG-110 has higher graphitization than NBG-18. This is consistent with the results in this study.

Polycrystalline graphite consists of filler particles and a binder phase. The high degree of graphitization of MG-based graphite is attributed to the MG filler particles. The degree of
Table 4 - CTE value and isotropy ratio of MG-based graphite, petroleum coke-based graphite, and IG-110.

\begin{tabular}{llll} 
& \multicolumn{2}{l}{$\mathrm{CTE}\left(10^{-6} \mathrm{~K}^{-1}, \mathrm{RT}-400^{\circ} \mathrm{C}\right)$} & Isotropy ratio \\
\cline { 2 - 3 } & $\mathrm{TR}$ & $\mathrm{AX}$ & \\
\hline MG & 2.81 & 3.14 & 1.12 \\
Coke & 3.44 & 4.73 & 1.37 \\
IG-110 & 4.06 & 4.35 & 1.07 \\
\hline
\end{tabular}

graphitization for MG ore from Lutang Chenzhou is $88.8 \%$, and increases to $91.7 \%$ after high temperature purification.

\subsubsection{Isotropy ratio and low CTE}

Isotropic ratio is another important parameter for nuclear graphite. Based on the ASTM standard [25], isotropic ratio is based on the CTE and is defined as the ratio of CTE in the against-grain direction to its corresponding value in the with-grain direction. For iso-molded graphite, the with-grain direction is defined as the against-gravity or transverse (TR) direction, whereas the against-grain direction is defined as the with-gravity or axial (AX) direction.

Nuclear graphite with high isotropy is very desirable for HTRs for two reasons. Firstly, isotropic graphite generally has a longer lifetime than anisotropic graphite. The dimensional change of isotropic and anisotropic graphite with dose is illustrated in Fig. 11. Nuclear graphite components are considered to reach their lifetime when the dimensional change in either the against-grain or with-grain direction returns to zero. For anisotropic graphite such as PGA graphite [22,26], the turnaround occurs at a much lower dose in the againstgrain direction, resulting in a short lifetime for the graphite. In contrast, isotropic graphite (such as Gilsocarbon graphite) shows similar dimensional stability in both directions $[22,27]$, thereby giving the graphite a much longer lifetime.

Secondly, anisotropic dimensional change may cause distortion or mismatch of graphite components, leading, for example, to interference with control rod operation or $\mathrm{He}$ leakage. As a result, nuclear graphite is required to be highly isotropic.

Fig. 12(a) and (b) shows CTE curves of MG-based and petroleum coke-based graphite, respectively, and a calibration run for IG-110 is shown in Fig. 12(c). Mean CTE values (30-

Table 5 - CTE values of some typical nuclear graphite.

\begin{tabular}{lll} 
& CTE $\left(10^{-6} \mathrm{~K}^{-1}\right)$ & Refs. \\
\hline GCMB Gilsocarbon & $/ / 4.99, \perp 4.84^{*}\left(20-120^{\circ} \mathrm{C}\right)$ & {$[28]$} \\
H-451 & $/ / 4.00, \perp 4.55$ & {$[4]$} \\
ATR-2E & $/ / 4.4, \perp 4.9$ & {$[29]$} \\
ASR-1SR & $/ / 4.70, \perp 4.87$ & {$[29]$} \\
PCEA & $/ / 4.0, \perp 4.24$ & {$[30]$} \\
IG-110 & $4.5\left(350-450^{\circ} \mathrm{C}\right)$ & Catalog value \\
& $4.06\left(20-400^{\circ} \mathrm{C}\right)$ & {$[31]$} \\
IG-430 & 4.8 & Catalog value \\
NBG-17 & $/ / 4.5, \perp 4.6$ & Catalog value \\
NBG-18 & $/ / 4.5, \perp 4.7$ & Catalog value \\
NBG-25 & $/ / 3.9, \perp 4.3$ & Catalog value \\
\hline " // means parallel to the forming axis (with-grain), $\perp$ means per- \\
pendicular to the forming axis (against-grain).
\end{tabular}


$400^{\circ} \mathrm{C}$ ) and the corresponding isotropy ratio are given in Table 4. The CTE values of IG-110 in the TR and AX directions are $4.06 \times 10^{-6}$ and $4.35 \times 10^{-6} \mathrm{~K}^{-1}$, respectively, in good agreement with Katoh's results. The MG-based graphite shows CTE values for the TR and AX directions that are close, giving rise to an isotropy ratio of 1.12. In contrast, coke-based graphite has an isotropy ratio of 1.37 , showing significantly anisotropic thermal expansion behavior.

The MG-based graphite shows CTE values of $2.81 \times 10^{-6}$ and $3.14 \times 10^{-6} \mathrm{~K}^{-1}$ in the TR and AX directions, respectively. Table 5 reports CTE values of some commercial nuclear graphites. It is of great interest to note that MG-based graphite has much lower CTE values than commercial nuclear graphites, which show CTE values in the range of 4.0$5.0 \times 10^{-6} \mathrm{~K}^{-1}$. Nuclear graphite with a low CTE gives rise to less thermal stress in the reactor core. A possible reason for the low CTE of MG-based graphite may be the unique microstructure of MG, which will be addressed in our future research.

\section{Concluding remarks}

The major conclusions of this work are as follows:

- The graphite micro-crystallites in MG show random orientation, leading to particles with weak anisotropy. After high temperature purification, the degree of graphitization for MG is more than $90 \%$.

- Green bodies produced from MG undergo a volume expansion in baking.

- MG-based graphite formed by isostatic pressing is nearisotropic. Furthermore, MG-based graphite is quite comparable in strength to graphite produced from petroleum coke.

- Benefits of MG-based graphite include high thermal diffusivity in green bodies, a high degree of graphitization, and a very low CTE value. These indicate a large potential for nuclear applications.

\section{Acknowledgments}

This work was supported by the National Natural Science Foundation of China (51402166) - China and China Postdoctoral Science Foundation (2014M550723). The authors would like to thank Sinosteel Advanced Materials (Zhejiang) Co. Ltd. for the help with impregnation and rebake. The authors would like to thank Dr. Burchell for discussions about the irradiation behavior of graphite.

\section{R E F E R E N C E S}

[1] Burchell TD. Radiation effects in graphite. In: Konings RJM, editor. Comprehensive nuclear materials. Amsterdam: Elsevier; 2012. p. 299-324.

[2] Windes WE, Burchell TD, Swank WD, Carroll MC. The next generation nuclear plant graphite R\&D program. Trans Am Nucl Soc 2011;105:187-8.
[3] Iyoku T, Shiozawa S, Ishihara M, Arai T, Oku T. Graphite core structures and their structural design criteria in the HTTR. Nucl Eng Des 1991;132(1):23-30.

[4] Burchell TD. Fission reactor application of carbon. In: Burchell TD, editor. Carbon materials for advanced technologies, Amsterdam. New York: Pergamon; 1999. p. 429-84.

[5] Xu SJ, Kang FY. Carbon and graphite materials in nuclear engineering. Beijing: Tsinghua University Press; 2010.

[6] Burchell TD, Snead LL. The effect of neutron irradiation damage on the properties of grade NBG-10 graphite. J Nucl Mater 2007;371(1-3):18-27.

[7] Marsden BJ, Hall GN. Graphite in gas-cooled reactors. In: Konings RJM, editor. Comprehensive nuclear materials. Amsterdam: Elsevier; 2012. p. 325-90.

[8] Mostafavi M, Schmidt MJJ, Marsden BJ, Marrow TJ. Fracture behaviour of an anisotropic polygranular graphite (PGA). Mater Sci Eng, A 2012;558:265-77.

[9] Laudone GM, Gribble CM, Matthews GP. Characterisation of the porous structure of Gilsocarbon graphite using pycnometry, cyclic porosimetry and void-network modeling. Carbon 2014;73:61-70.

[10] Zou L, Kang FY, Li XH, Zheng YP, Shen WC, Zhang J. Investigations on the modified natural graphite as anode materials in lithium ion battery. J Phys Chem Solids 2008;69(5-6):1265-71.

[11] Zou L, Kang FY, Zheng YP, Shen WC. Modified natural flake graphite with high cycle performance as anode material in lithium ion batteries. Electrochim Acta 2009;54(15):3930-4.

[12] Bäumer R, Energietechnik V-G. AVR, experimental hightemperature reactor: 21 years of successful operation for a future energy technology. VDI-Verlag; 1990.

[13] Zhao HS, Liang TX, Zhang J, He J, Zou YW, Tang CH. Manufacture and characteristics of spherical fuel elements for the HTR-10. Nucl Eng Des 2006;236(5-6):643-7.

[14] Parker WJ, Jenkins RJ, Abbott GL, Butler CP. Flash method of determining thermal diffusivity, heat capacity, and thermal conductivity. J Appl Phys 1961;32(9):1679-84.

[15] Vozar L, Hohenauer W. Flash method of measuring the thermal diffusivity. A review. High Temp - High Press 2003;35-6(3):253-64.

[16] ASTM. Standard test method for thermal diffusivity by the flash method. Philadelphia: American Society for Testing and Materials; 2008.

[17] Tron AR. The production and uses of natural graphite. London: Her Majesty's Stationery Office; 1964.

[18] Lewis IC. Chemistry of pitch carbonization. Fuel 1987;66(11):1527-31.

[19] Neighbour GB. Modelling of dimensional changes in irradiated nuclear graphites. J Phys D-Appl Phys 2000;33(22):2966-72.

[20] Kelly BT. Irradiation behavior of fine-grain graphites. Fusion Technol 1989;16(1):96-103.

[21] Oku T, Ishihara M. Lifetime evaluation of graphite components for HTGRs. Nucl Eng Des 2004;227(2):209-17.

[22] Brocklehurst JE, Kelly BT. Analysis of the dimensional changes and structural-changes in polycrystalline graphite under fast-neutron irradiation. Carbon 1993;31(1): 155-78.

[23] Zheng GQ, Xu P, Sridharan K, Allen T. Characterization of structural defects in nuclear graphite IG-110 and NBG-18. J Nucl Mater 2014;446(1-3):193-9.

[24] Chi SH, Kim GC. Comparison of the oxidation rate and degree of graphitization of selected IG and NBG nuclear graphite grades. J Nucl Mater 2008;381(1-2):9-14.

[25] ASTM. Standard specification for isotropic and near-isotropic nuclear graphites. Philadelphia: American Society for Testing and Materials; 2008. 
[26] Kelly BT, Martin WH, Nettley PT. Dimensional changes in polycrystalline graphites under fast-neutron irradiation. Philos Trans R Soc London Ser A 1966;260(1109):51-71.

[27] Marsden BJ, Hall GN, Wouters O, Vreeling JA, van der Laan J. Dimensional and material property changes to irradiated Gilsocarbon graphite irradiated between 650 and 750 C. J Nucl Mater 2008;381(1-2):62-7.

[28] Preston SD, Marsden BJ. Changes in the coefficient of thermal expansion in stressed Gilsocarbon graphite. Carbon 2006;44(7):1250-7.
[29] Haag G, Mindermann D, Wilhelmi G, Persicke H, Ulsamer W. Development of reactor graphite. J Nucl Mater 1990;171(1):41-8.

[30] El-Genk MS, Tournier JMP. Comparison of oxidation model predictions with gasification data of IG-110, IG-430 and NBG-25 nuclear graphite. J Nucl Mater 2012;420(1-3): 141-58.

[31] Ishihara M, Iyoky T, Takikawa N, Shiozawa S. Component test of the core support post of the HTTR. Vienna: IAEA; 1993. 